\title{
Concomitant Radiation Recall Dermatitis and Organizing Pneumonia following Breast Radiotherapy: A Case Report
}

\author{
I-Chia Liu ${ }^{a}$ Fantine Giap ${ }^{a} \quad$ Raymond B. Mailhot-Vega ${ }^{a} \quad$ Julie A. Bradley ${ }^{a}$ \\ Nancy P. Mendenhall ${ }^{a}$ Paul Okunieffa ${ }^{a}$ Li Lu $^{b}$ Michael A. Jantz ${ }^{c}$ \\ Karen Dailyc ${ }^{c}$ Lisa Spiguel ${ }^{d}$ Natalie A. Lockney ${ }^{a}$ \\ aDepartment of Radiation Oncology, University of Florida College of Medicine, Gainesville, \\ FL, USA; bepartment of Pathology, University of Florida College of Medicine, Gainesville, \\ FL, USA; 'Department of Medicine, University of Florida College of Medicine, Gainesville, FL, \\ USA; ${ }^{d}$ Department of Surgery, University of Florida College of Medicine, Gainesville, FL, USA
}

\section{Keywords}

Radiation toxicity $\cdot$ Radiation recall $\cdot$ Dermatitis $\cdot$ Radiation-induced organizing pneumonia

\begin{abstract}
Purpose: Radiation recall dermatitis (RRD) is a rare complication that occurs after completion of radiation therapy (RT) and initiation of a precipitating agent, most commonly chemotherapeutic medications. Various theories attempt to explain the mechanism, including activation of the body's inflammatory pathways through nonimmune activation. Likewise, radiation-induced organizing pneumonia (RIOP) is an infrequent but potentially life-threatening complication of RT that, while not fully understood, is suspected to be partly an autoimmune reaction. Patient: We present the case of a 71-year-old female with a history of type 2 diabetes mellitus, hypothyroidism, interstitial cystitis, and osteoarthritis who presented with clinical stage T1N0M0 ER+/PR-/HER2- invasive ductal carcinoma of the lower outer quadrant of the left breast, for which she underwent left segmental mastectomy and sentinel lymph node biopsy followed by completion axillary lymph node dissection. Her final pathologic stage was T1N1M0. Result: The patient developed RRD and later RIOP following receipt of radiation and chemotherapy, which resolved with steroid administration. Conclusions: The rarity of both RRD and RIOP occurring in a patient, as in our case, suggests a shared pathophysiology behind these two complications. As both reactions involve some degree of inflammation and respond to corticosteroids, it seems likely that the etiologies of RRD and RIOP lie within the inflammatory pathway. However, further investigation should evaluate the frequency, duration, and triggering of concomitant RRD and RIOP.




\section{Introduction}

Radiation recall dermatitis (RRD) is a rare but potentially severe adverse effect of radiation therapy (RT) that occurs after radiation and is triggered by systemic agents. One hypothesis explains RRD as an inflammatory response that is first initiated by RT and later exacerbated by chemotherapeutic agents [1]. Multiple drugs (sorafenib, docetaxel, and tamoxifen) have been identified as culprits behind RRD in various case reports [2-4], but the exact mechanism for this delayed development of dermatitis remains unclear. Radiationinduced organizing pneumonia (RIOP) is another uncommon complication of RT [5] that can have significant clinical consequences. RIOP appears to be caused by an inflammatory component as well [6]. However, the relationship between RRD and other inflammatory and delayed adverse effects of RT, including lung injuries such as RIOP, have yet to be fully understood.

Herein, we present a patient with early-stage breast cancer who developed RRD and later RIOP after receiving RT and chemotherapy, and review the literature regarding these rare complications.

\section{Case Presentation}

A 71-year-old female with a history of type 2 diabetes mellitus, hypothyroidism, interstitial cystitis, and osteoarthritis presented with clinical stage T1N0M0 ER+/PR-/HER2invasive ductal carcinoma of the lower outer quadrant of the left breast, for which she underwent left segmental mastectomy and sentinel lymph node biopsy. Pathology demonstrated a 14-mm grade 2 invasive ductal carcinoma with ductal carcinoma in situ, positive lymphovascular invasion, and ductal carcinoma in situ present at the margins. One of 3 sentinel lymph nodes was positive with $8-\mathrm{mm}$ macrometastasis and $4.5-\mathrm{mm}$ extracapsular extension that was not present on gross examination. She then underwent re-excision of the breast with negative final margins and completion axillary lymph node dissection, which identified 13 lymph nodes, all negative for carcinoma. Oncotype testing was ordered, but processing issues occurred, resulting in a delay. The decision was thus made to proceed with adjuvant radiotherapy within 8 weeks of the surgery date.

RT was delivered to the whole left breast using active breathing control to a dose of 42.4 Gy in 16 fractions with opposed photon tangents with a field-in-field technique to minimize dose heterogeneity using 6- and 8-MV photons delivered by a linear accelerator. The patient received a sequential tumor bed boost without active breathing control to a dose of $10 \mathrm{~Gy}$ in 4 fractions using a 3-dimensional conformal technique with 6-MV photons. The ipsilateral lung $\mathrm{V}_{4 \mathrm{~Gy}}, \mathrm{~V}_{16 \mathrm{~Gy}}$, and $\mathrm{V}_{20 \mathrm{~Gy}}$ were 27,14 , and $12 \%$, respectively, and the mean heart dose was $1.04 \mathrm{~Gy}$. During treatment, the patient complained of intermittent burning and mild discomfort in the breast, alleviated by daily acetaminophen. Mild skin erythema was noted, and the patient applied Miaderm twice daily to her breast during treatment. She completed RT without interruptions; no desquamation was observed at the last on-treatment visit.

After completing radiation, the patient's OncotypeDX returned with a score of 31, for which she was advised to pursue adjuvant chemotherapy. Docetaxel and cyclophosphamide were initiated 3 weeks after RT completion and planned for 4 cycles. Prior to initiation of chemotherapy, her skin was intact with erythema limited to the axilla. She then presented with left breast tenderness, erythema, and warmth with no fevers 1 week after starting chemotherapy and 4 weeks after completing RT (Fig. 1A, B). At the time of physical examination, the differential diagnosis included cellulitis versus RRD, and a trial of cephalexin for 1 week was prescribed. After no improvement, she was prescribed oral prednisone at $20 \mathrm{mg}$ 
A
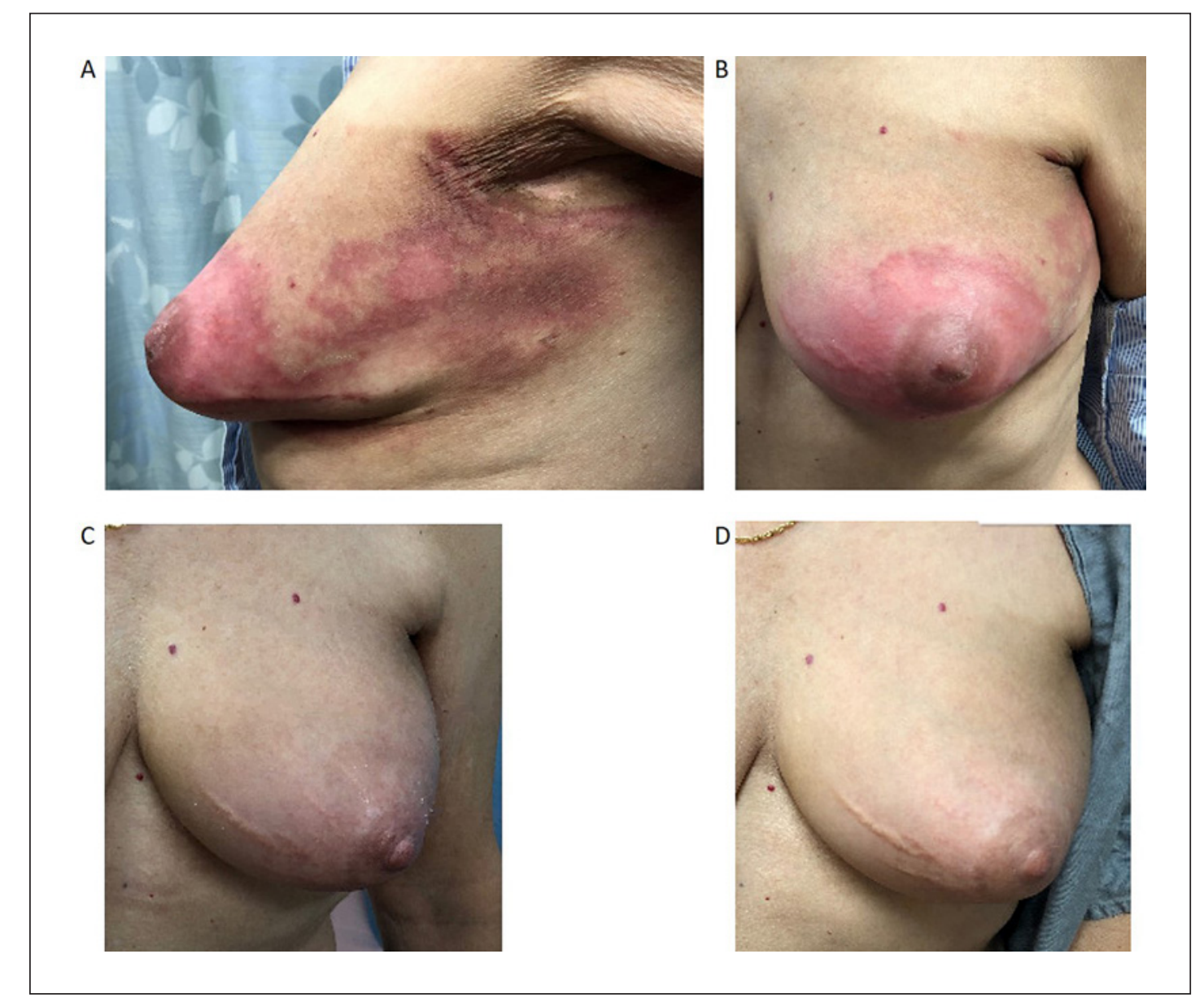

C

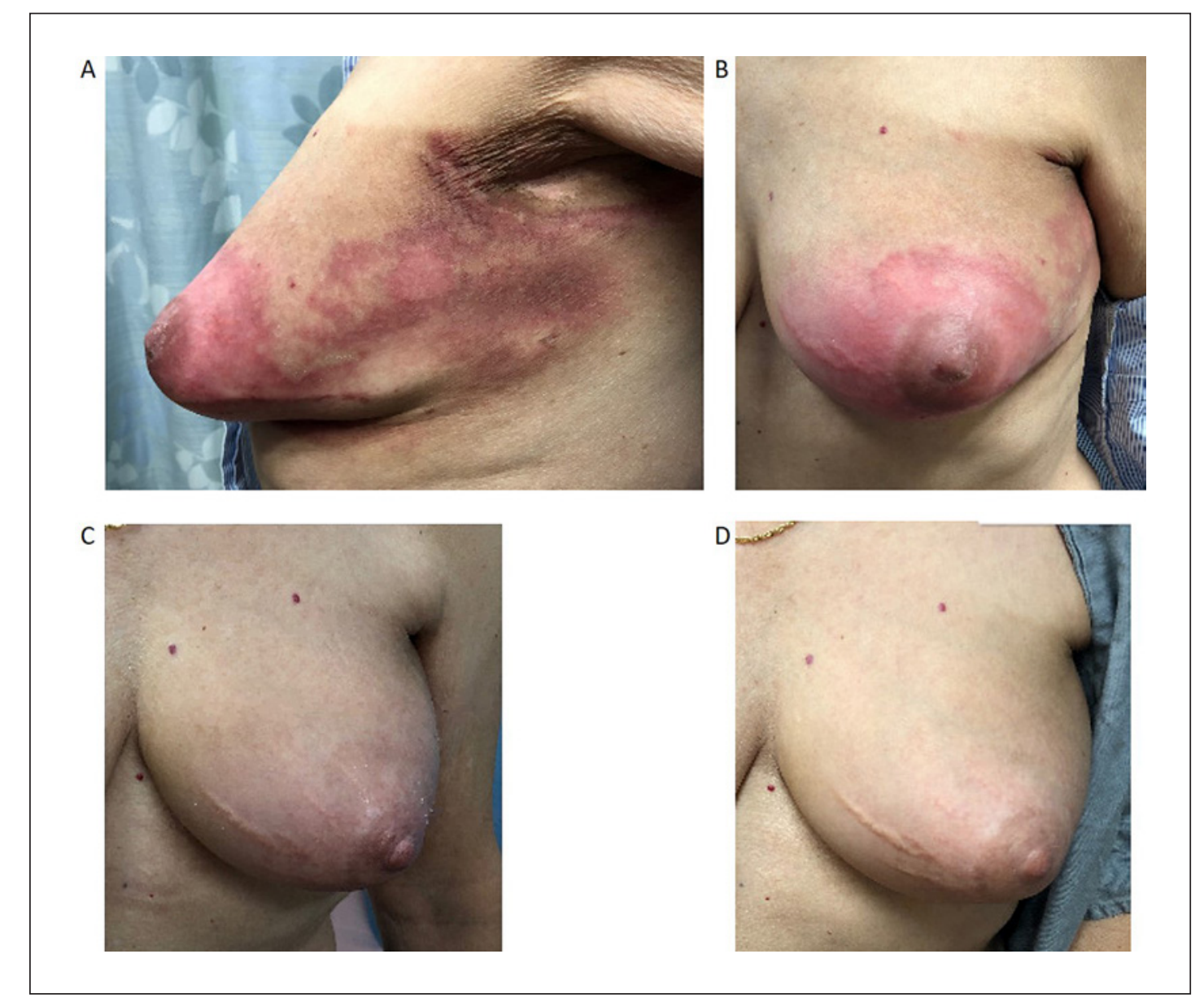

(c) 2020 The Author(s). Published by S. Karger AG, Basel www.karger.com/cro

Liu et al.: Radiation Recall Dermatitis and Organizing Pneumonia

Fig. 1. Representative photographs of the left breast during clinical examination at various time points. A, B Four weeks after radiation therapy and 1 week after chemotherapy. C One week after prednisone initiation. D One week after letrozole initiation.

daily for 7 days and silver sulfadiazine for presumed RRD. The patient returned to the clinic 1 week after starting prednisone therapy and reported marked improvement in her skin appearance (Fig. 1)C. She was premedicated with $20 \mathrm{mg}$ of prednisone for cycle 2 of chemotherapy. However, she developed less severe RRD after her second cycle of chemotherapy, so prednisone was increased to $30 \mathrm{mg}$, followed by a brief taper for the third and fourth chemotherapy cycles. The patient tolerated the remaining chemotherapy cycles well and successfully completed chemotherapy.

When the patient began letrozole 3 weeks after having completed all chemotherapy, she reported breast warmth similar to what she had experienced before developing RRD, although the clinical examination was unremarkable (Fig. 1D). After discussion with a medical oncologist, letrozole was held for 2-3 weeks. As there were no changes to the breast during this time, letrozole was reinitiated. Her breast warmth and heaviness were attributed to breast lymphedema, and she continued physical therapy management.

At her 6-month post-radiation treatment follow-up, she reported having had a dry cough for 2 weeks without fever; a chest X-ray revealed consolidation in the left middle lung concerning for pneumonia. Of note, she had a computed tomography (CT) chest scan performed 1 month prior to radiation as part of a research study, which had shown postra- 
Fig. 2. Representative axial computed tomography images of the chest at various time points. A Five months after completion of radiation therapy (RT). B Six months after completion of RT. C Approximately 7 months after completion of RT. D-F 9.5 months after completion of RT. G-I Approximately 1 year after completing RT.

Fig. 3. A transbronchial biopsy specimen from a right lung lesion obtained 9.5 months after completion of radiation therapy (H\&E stain; original magnification, $\times 200$ ) demonstrated organizing pneumonia with no evidence of carcinoma.
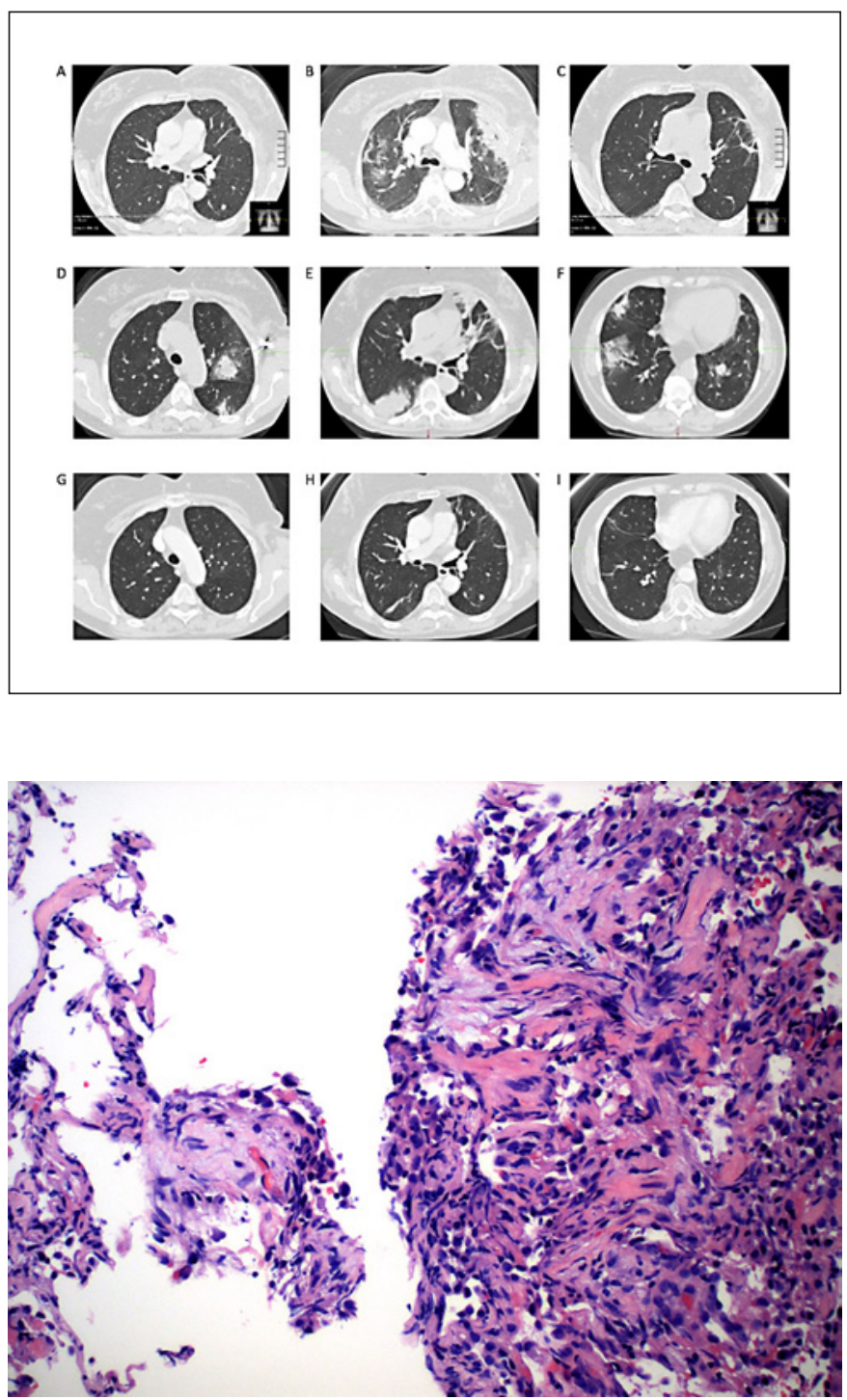

diation changes (Fig. 2A). For her symptoms and abnormal X-ray, she was prescribed azithromycin and benzonatate. However, she then became febrile to $101.5^{\circ} \mathrm{F}$ and presented to the emergency department, where a CT angiogram of her chest showed evidence of left upperand left lower-lobe infiltrates (Fig. 2B). She was admitted for intravenous antibiotics but showed no improvement and developed increased shortness of breath. Bronchoscopy with bronchoalveolar lavage revealed elevated lymphocytes (73\%), monocytes (2\%), and eosinophils $(12 \%)$ on the cell count differential, and cultures showed only normal respiratory flora. The patient was thus initiated on prednisone at $60 \mathrm{mg}$ daily for presumed radiation pneumonitis; she reported an improvement in symptoms within 1 day. A chest CT completed around 1.5 months later demonstrated improvement in the consolidation, and the patient had returned to her baseline respiratory function clinically and on pulmonary function tests (Fig. 2C). She completed prednisone tapering approximately 1 month later.

The patient was admitted again to the hospital 1.5 months after completing the steroid taper (9.5 months after completing RT) due to fatigue and shortness of breath. A chest CT showed bilateral multifocal rounded consolidations and airspace disease concerning for 
organizing pneumonia (Fig. 2D-F). Bronchoscopy with bronchoalveolar lavage showed 2\% lymphocytes, $81 \%$ monocytes, and 3\% eosinophils. Cultures revealed only normal respiratory flora. A biopsy of the right lung revealed organizing pneumonia (Fig. 3) with stains negative for fungal organisms or pneumocystis. She briefly required supplemental oxygen after a bronchoscopy but was discharged without oxygen requirement. She was placed on 60 mg prednisone following the biopsy results and prophylactic sulfamethoxazole/trimethoprim with pulmonological follow-up. Two months later, she reported no shortness of breath and only occasional cough on $30 \mathrm{mg}$ prednisone, with a chest CT demonstrating resolution of the prior airspace consolidation (Fig. 2G-I). She did report side effects from the steroids, including insomnia, a 16-lb weight gain over the previous 2 months, and elevated blood glucose levels. She was advised to complete a steroid taper over the next 2 months with further follow-up.

\section{Discussion}

RRD remains a conundrum, with no definitive standard of care established to prevent and treat this potentially serious complication. Its incidence is difficult to report as the literature is primarily limited to single cases, with some small case series reporting an incidence as high as $8-11 \%[7,8]$. Current theories of mechanism include stem cell malfunction in the irradiated area [9], localized hypersensitivity that activates nonimmune inflammatory pathways [1], and keratinocyte necrosis [10]. Although taxanes and anthracyclines have been identified as major contributors to RRD [11], a variety of agents, including nonchemotherapeutic medications, have been identified as triggers of this reaction: actinomycin D, doxorubicin, levofloxacin [12], antituberculosis agents, and simvastatin. According to two case reports, RRD occurs more often in areas that receive higher radiation doses $[13,14]$, but the dose range reported to cause RRD varies widely, as illustrated in reviews by Burris and Hurtig [15] and Camidge and Price [1], who reviewed reports with doses as low as 10 Gy (range, 10-61.2). Moreover, RRD can occur over a wide timespan. Camidge and Price [1] reported a range of 7-840 days (median, 39.5) between RT and chemotherapy among patients who experienced RRD. In one case, RRD occurred 25 years after RT [16]. Initiating chemotherapy at least 3 weeks after completing RT was suggested based on a demonstrated (yet statistically nonsignificant) reduction in the rate of patients who develop RRD following chemotherapy $>3$ weeks after breast MammoSite RT (7.4\%) compared to $\leq 3$ weeks after RT $(18 \%)$ in the Breast Surgeons MammoSite Breast Brachytherapy Registry Trial [8]. Depending on the severity of symptoms, treatment options range from observation alone to the use of systemic or topical steroids or nonsteroidal anti-inflammatory drugs to decrease inflammation [15]. While discontinuing the offending agent should lead to an improvement in symptoms, the question of recurrence of RRD after rechallenging with the same offending agent, or an agent of the same class, remains unclear $[11,15]$.

Whether RRD is associated with an increased incidence of late radiation side effects is unknown. It often occurs in patients with minimal or no acute radiation toxicity, such as with our patient. Our patient also experienced the relatively uncommon complication of radiationinduced lung injury following breast RT, however, suggesting that those with RRD (or those who receive chemotherapy after radiation) may be predisposed to late radiation-induced complications. It is unknown if concurrent inflammatory changes were occurring in the lung simultaneously to the clinically apparent dermatitis, since lung imaging was not indicated at that time in the absence of respiratory symptoms. To our knowledge, concurrent RRD and RIOP has been reported in only 2 cases in the literature $[17,18]$, although the incidence may be underreported, since dermatitis is more clinically apparent than pneumonitis unless imaging is obtained in symptomatic patients. In modern studies, grade 2 or higher radiation 
pneumonitis or lung fibrosis is uncommon (0.3\%) following whole-breast RT [19]. The lung dose in this case was low and met standard institutional and cooperative group guideline criteria [20].

Initially, our patient was thought to have radiation pneumonitis; however, when she later developed contralateral lesions in the right lung and underwent lung biopsy, organizing pneumonia - also referred to as bronchiolitis obliterans organizing pneumonia - became the leading diagnosis. As explained by Oie et al. [21], radiation pneumonitis only occurs in regions that have received radiation, but RIOP lesions can migrate away from the originally radiated regions. Oie et al. [21] reported an organizing pneumonia rate of $1.2 \%$ among patients receiving RT for breast cancer to the breast or chest wall \pm regional lymph nodes. Other studies have shown the incidence of RIOP to be $<3 \%$ in patients receiving radiation to the breast $[5,6,22]$.

The development of RIOP has been attributed to interactions between radiotherapy and the immune system [23]. Organizing pneumonia has also been reported, however, in cancer patients after chemotherapy, targeted therapy, or immunotherapy as a manifestation of druginduced lung injury in the absence of radiation [24-26]. Thus, organizing pneumonia is not a phenomenon limited to patients receiving radiation. Interestingly, Katayama etal. [5] reported that endocrine therapy concurrent with radiation was associated with an increased incidence of RIOP, which is supported in part by other studies showing that tamoxifen given concurrently with postmastectomy radiation is associated with increased pulmonary fibrosis [27, 28]. Age also appears to be implicated as a potential factor in the development of bronchiolitis obliterans organizing pneumonia [5] and pneumonitis [29, 30]. Yet, other studies have reported no associations between RIOP and antiestrogen medications [31], tamoxifen, chemotherapy, or patient age [6]. While the treatment for RIOP is corticosteroids, which can provide rapid relief of symptoms, interestingly, corticosteroids were associated with an increased relapse rate of RIOP compared to nonsteroidal medications, such as nonsteroidal anti-inflammatory drugs, antibiotics, or antitussives, in a single-institution retrospective study [22]. Lung injury from chemotherapy, RT, or radiation recall should not be taken lightly, as severe cases can lead to death [32].

Both RRD and RIOP are uncommon adverse effects from RT, and observing them concurrently suggests a relationship rather than a coincidence. Our case is unique, with only 2 other cases of concurrent RRD and RIOP available in the literature $[15,16]$. As alluded to earlier, one theory explaining RRD is nonimmune activation of inflammatory pathways, which is comparable to fixed drug eruptions [1]. Radiation can increase the production of inflammatory cytokines and, when a chemotherapeutic agent is initiated, the number of inflammatory cytokines may increase, leading to the symptoms seen in patients with radiation recall. Similarly, RIOP is an inflammatory response to tissue injury [33], but it appears that an immunologic reaction is involved with the increase in eosinophils, lymphocytes, and neutrophils [6]. Inflammatory monocytes have been shown to migrate into the lung following radiation exposure and are implicated in the pathogenesis of radiation-induced lung injury [34], and our patient had a spike in monocytes at the time of her RIOP diagnosis. The potential relationship between RRD, RIOP, and dysregulation of the body's immune pathways requires further study.

\section{Conclusions}

In the present report, we discussed the case of a 71-year-old female with breast cancer who developed both RRD and RIOP after receiving RT and chemotherapy, a rare combination of complications documented in very few case reports. Although both adverse events can

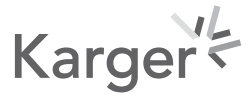


resolve over time with corticosteroids and symptomatic management, they must be monitored as there are no accurate predictors for their occurrence and they may be misdiagnosed. RRD and RIOP are both uncommon phenomena, so the chance of them occurring unrelatedly seems unlikely. Since both reactions involve some degree of inflammation and respond to corticosteroids, the etiologies of RRD and RIOP likely exist within the inflammatory pathway. Yet, further investigations should be pursued to evaluate the frequency, duration, and causes of concomitant RRD and RIO.

\section{Acknowledgments}

We would like to thank Jessica Kirwan and Christopher Stich for editorial assistance.

\section{Statement of Ethics}

All patients provided written informed consent to publish their case data and images.

\section{Conflict of Interest Statement}

The authors have nothing to disclose.

\section{Funding Sources}

No funding was received.

\section{Author Contributions}

I.-C.L.: collecting data, analyzing data, and writing the manuscript; F.G: collecting data, analyzing data, and revising the manuscript; R.B.M.-V.: analyzing data and writing the manuscript; J.A.B., N.P.M., P.O., L.L., M.A.J., and L.S.: revising the manuscript; N.A.L.: conceptualizing the manuscript, collecting data, and writing the manuscript.

\section{References}

1 Camidge R, Price A. Characterizing the phenomenon of radiation recall dermatitis. Radiother Oncol. 2001; 59(3):237-45.

2 Bourgeois A, Grisoli SB, Soine EJ, Rosen LB. Tamoxifen-induced radiation recall dermatitis. Dermatol Online J. 2017;23(2):13030/qt1d38c9c7.

3 Kim GE, Song HS, Ahn KJ, Kim YS. Radiation recall dermatitis triggered by sorafenib after radiation therapy for hepatocellular carcinoma. Radiat Oncol J. 2017;35(3):289-94.

4 Sakaguchi M, Maebayashi T, Aizawa T, Ishibashi N. Docetaxel-induced radiation recall dermatitis with atypical features: a case report. Medicine (Baltimore). 2018;97(36):e12209.

5 Katayama N, Sato S, Katsui K, Takemoto M, Tsuda T, Yoshida A, et al. Analysis of factors associated with radiation-induced bronchiolitis obliterans organizing pneumonia syndrome after breast-conserving therapy. Int J Radiat Oncol Biol Phys. 2009;73(4):1049-54.

6 Takigawa N, Segawa Y, Saeki T, Kataoka M, Ida M, Kishino D, et al. Bronchiolitis obliterans organizing pneumonia syndrome in breast-conserving therapy for early breast cancer: radiation-induced lung toxicity. Int J Radiat Oncol Biol Phys. 2000;48(3):751-5. 
7 Kodym E, Kalinska R, Ehringfeld C, Sterbik-Lamina A, Kodym R, Hohenberg G. Frequency of radiation recall dermatitis in adult cancer patients. Onkologie. 2005;28(1):18-21.

8 Haffty BG, Vicini FA, Beitsch P, Quiet C, Keleher A, Garcia D, et al. Timing of chemotherapy after MammoSite radiation therapy system breast brachytherapy: analysis of the American Society of Breast Surgeons MammoSite Breast Brachytherapy Registry Trial. Int J Radiat Oncol Biol Phys. 2008;72(5):1441-8.

9 Hellman S, Botnick LE. Stem cell depletion: an explanation of the late effects of cytotoxins. Int J Radiat Oncol Biol Phys. 1977;2(1-2):181-4.

10 Smith KJ, Germain M, Skelton H. Histopathologic features seen with radiation recall or enhancement eruptions. J Cutan Med Surg. 2002;6(6):535-40.

11 Azria D, Magné N, Zouhair A, Castadot P, Culine S, Ychou M, et al. Radiation recall: a well recognized but neglected phenomenon. Cancer Treat Rev. 2005;31(7):555-70.

12 Wernicke AG, Swistel AJ, Parashar B, Myskowski PL. Levofloxacin-induced radiation recall dermatitis: a case report and a review of the literature. Clin Breast Cancer. 2010;10(5):404-6.

13 Yeo W, Leung SF, Johnson PJ. Radiation-recall dermatitis with docetaxel: establishment of a requisite radiation threshold. Eur J Cancer. 1997;33(4):698-9.

14 Stelzer KJ, Griffin TW, Koh WJ. Radiation recall skin toxicity with bleomycin in a patient with Kaposi sarcoma related to acquired immune deficiency syndrome. Cancer. 1993;71(4):1322-5.

15 Burris HA, Hurtig J. Radiation recall with anticancer agents. Oncologist. 2010;15(11):1227-37.

16 Barlési F, Tummino C, Tasei AM, Astoul P. Unsuccessful rechallenge with pemetrexed after a previous radiation recall dermatitis. Lung Cancer. 2006;54(3):423-5.

17 Lee HE, Jeong NJ, Lee Y, Seo YJ, Kim CD, Lee JH, et al. Radiation recall dermatitis and pneumonitis induced by trastuzumab (Herceptin®). Int J Dermatol. 2014;53(3):e159-60.

18 Schweitzer VG, Juillard GJ, Bajada CL, Parker RG. Radiation recall dermatitis and pneumonitis in a patient treated with paclitaxel. Cancer. 1995;76(6):1069-72.

19 Whelan TJ, Olivotto IA, Parulekar WR, Ackerman I, Chua BH, Nabid A, et al. Regional nodal irradiation in earlystage breast cancer. N Engl J Med. 2015;373(4):307-16.

20 NRG Oncology. RTOG 1005: a phase III trial of accelerated whole breast irradiation with hypofractionation plus concurrent boost versus standard whole breast irradiation plus sequential boost for early-stage breast cancer. 2014 [accessed 2020 Jan 20]. Available from: https://www.rtog.org/ClinicalTrials/ProtocolTable/ StudyDetails.aspx?action = openFile\&FileID $=9366$.

21 Oie Y, Saito Y, Kato M, Ito F, Hattori H, Toyama H, et al. Relationship between radiation pneumonitis and organizing pneumonia after radiotherapy for breast cancer. Radiat Oncol. 2013;8:56.

22 Otani K, Nishiyama K, Ito Y, Kawaguchi Y, Inaji H. Steroid treatment increases the recurrence of radiationinduced organizing pneumonia after breast-conserving therapy. Cancer Med. 2014;3(4):947-53.

23 Ogo E, Komaki R, Fujimoto K, Uchida M, Abe T, Nakamura K, et al. A survey of radiation-induced bronchiolitis obliterans organizing pneumonia syndrome after breast-conserving therapy in Japan. Int J Radiat Oncol Biol Phys. 2008;71(1):123-31.

24 Comito F, Grassi E, Poerio A, Freier E, Calculli L, Zompatori M, et al. Organizing pneumonia after pancreatic cancer treatment with nab-paclitaxel and gemcitabine: a case report. BJR Case Rep. 2018;4(2):20170086.

25 Lim SM, An HJ, Park HS, Kwon HJ, Kim EY, Hur J, et al. Organizing pneumonia resembling disease progression in a non-small-cell lung cancer patient receiving ceritinib: a case report. Medicine (Baltimore). 2018;97(31): e11646.

26 Kashiwada T, Minegishi Y, Saito Y, Kato T, Atsumi K, Seike M, et al. Organizing pneumonia after nivolumab treatment in a patient with pathologically proven idiopathic pulmonary fibrosis. J Nippon Med Sch. 2019; 86(1):43-7.

27 Koc M, Polat P, Suma S. Effects of tamoxifen on pulmonary fibrosis after cobalt-60 radiotherapy in breast cancer patients. Radiother Oncol. 2002;64(2):171-5.

28 Bentzen SM, Skoczylas JZ, Overgaard M, Overgaard J. Radiotherapy-related lung fibrosis enhanced by tamoxifen. J Natl Cancer Inst. 1996;88(13):918-22.

29 Lind PA, Wennberg B, Gagliardi G, Rosfors S, Blom-Goldman U, Lideståhl A, et al. ROC curves and evaluation of radiation-induced pulmonary toxicity in breast cancer. Int J Radiat Oncol Biol Phys. 2006;64(3):765-70.

30 Dörr W, Bertmann S, Herrmann T. Radiation induced lung reactions in breast cancer therapy. Modulating factors and consequential effects. Strahlenther Onkol. 2005;181(9):567-73.

31 Kubo A, Osaki K, Kawanaka T, Furutani S, Ikushima H, Nishitani H. Risk factors for radiation pneumonitis caused by whole breast irradiation following breast-conserving surgery. J Med Invest. 2009;56(3-4):99-110.

32 Bargagli E, Bonti V, Bindi A, Scotti V, Pistolesi M, Voltolini L, et al. Fibrotic lung toxicity induced by cytotoxic drugs, radiation and immunotherapy in patients treated for lung cancer. Monaldi Arch Chest Dis. 2018;88(2): 917.

33 Otani K, Seo Y, Ogawa K. Radiation-induced organizing pneumonia: a characteristic disease that requires symptom-oriented management. Int J Mol Sci. 2017;18(2):281.

34 Groves AM, Johnston CJ, Williams JP, Finkelstein JN. Role of infiltrating monocytes in the development of radiation-induced pulmonary fibrosis. Radiat Res. 2018;189(3):300-11. 